\title{
The importance of knowledge in vivid text memory: An individual-differences investigation of recollection and familiarity
}

\author{
DEBRA L. LONG \\ University of California, Davis, California \\ and University of Central Lancashire, Preston, England \\ Chantel Prat \\ Carnegie Mellon University, Pittsburgh, Pennsylvania
}

AND

\author{
Clinton Johns, Phillip Morris, and Eunike Jonathan \\ University of California, Davis, California
}

\begin{abstract}
The goal of this study was to examine how individual variation in readers' skills and, in particular, their background knowledge about a text are related to text memory. Recollection and familiarity estimates were obtained from remember and know judgments to text ideas. Recollection estimates to old items were predicted by readers' background knowledge, but not by other comprehension-related factors, such as word-decoding skill and working memory capacity. False alarms involving recollection of new items (inferences) were diminished as a function of verbal ability, working memory capacity, and reasoning but increased as a function of background knowledge. The results suggest that recollection indexes the reader's ability to construct a text representation in which text ideas are integrated with relevant domain knowledge. Moreover, these results highlight the importance of background knowledge in explaining individual variation in comprehension and memory for text.
\end{abstract}

Readers who understand a text construct a representation in which explicit text ideas are integrated with each other and with relevant background knowledge. This representation is often called a discourse model, a model of the real or imaginary world that the text describes (Gernsbacher, 1990; Kintsch, 1988). Readers use their discourse model to perform a variety of activities, including recall, recognition, and question-answering.

One of the earliest findings in the study of text comprehension was that readers who have background knowledge about a text remember it better than do readers who lack such knowledge (Bransford \& Johnson, 1972). The memory advantage associated with prior knowledge, however, is generally limited to recall (Anderson \& Pichert, 1978; Bower, Black, \& Turner, 1979; Johnson \& Kieras, 1983; Sulin \& Dooling, 1974). Studies investigating recognition of text ideas either have shown no knowledge effects or have shown that knowledge influences recognition of distractor sentences, such as inferences that may be made during comprehension (Alba \& Hasher, 1983; Moravcsik \& Kintsch, 1993).

Recognition appears to be insensitive to the influence of background knowledge because the task is too easy. Most readers, even those who lack prior knowledge, can readily discriminate sentences that appeared in a text from those that did not appear. Some researchers have argued that test sentences in recognition provide direct access to memory traces, eliminating the need to engage in an elaborate search of the text representation. Knowledge effects are found in recall, but not recognition, because knowledge primarily affects the search process (Moravcsik \& Kintsch, 1993).

Long and her colleagues have recently revisited the use of recognition to investigate text memory (Long \& Prat, 2002; Long, Wilson, Hurley, \& Prat, 2006). They argue that qualitative differences in memory for text ideas may result from differences in the processes that are involved in constructing sentence representations and those that are involved in constructing discourse models. Their claim is founded in dual-process models of memory in which two distinct processes support recognition.

\section{Dual-Process Models of Recognition Memory}

Dual-process models propose that recognition is the product of two memory processes: recollection and familiarity (e.g., Jacoby \& Dallas, 1981; Mandler, 1980; Yonelinas, 2002). Recollection is a relatively slow process, similar to recall, and yields qualitative information

D. L. Long, dllong@ucdavis.edu 
about studied events (e.g., when or where an item was studied). In some models, recollection is conceptualized as a threshold process, involving all-or-none retrieval of contextual information that is associated with a studied item. Familiarity is often conceptualized as a signal detection process, involving a quantitative assessment of the similarity (perceptual and conceptual) between a test item and a memory trace.

Several methods are used to estimate recollection and familiarity. The method used here is the remember/know task (e.g., Tulving, 1985). Participants make judgments about the nature of their memory for recognized items, responding remember if items are accompanied by retrieval of episodic details about their prior occurrence and responding know if items are recognized but are unaccompanied by remembering. Remember judgments are used as an index of recollection. Familiarity is estimated by the conditional probability that an item received a know response given that it was not recollected (Yonelinas \& Jacoby, 1995).

The remember/know task has been used extensively to examine factors that differentially affect recollection and familiarity (for a review, see Yonelinas, 2002). For example, recollection is strongly influenced by elaborative encoding, typically involving levels-of-processing manipulations. Recollection is also positively correlated with attention during both encoding and retrieval. Familiarity, in contrast, is sensitive to perceptual changes between study and test and is influenced by fluency manipulations, such as subliminal masked priming. Familiarity is also more affected by manipulations of response bias than is recollection.

\section{Using Recognition to Assess the Discourse Model}

Long and her colleagues have used dual-process models as a framework for understanding how text representations support memory (Long \& Prat, 2002; Long, Wilson, et al., 2006). When readers encounter sentences in texts, they execute orthographic, syntactic, and semantic processes to extract text ideas. These processes result in relatively rich item representations, akin to those that are constructed in the "deep" processing conditions involving levels-ofprocessing manipulations in the study memory for word lists. Sentence-level processes alone, however, do not yield coherent discourse models - that is, models of the text as a whole. Rather, they produce "list-like" representations, in which text ideas are represented individually or in clusters, loosely linked by theme (Long, Oppy, \& Seely, 1994, 1997). These representations support recognition, but they do so primarily by means of familiarity.

When readers construct an integrated discourse model of the entire text, however, they form a network of connections integrating text ideas with each other and with relevant knowledge. The discourse model also supports recognition, but it does so via recollection. A test sentence accesses its item representation in memory and activates the network of contextual information that was constructed during comprehension. Retrieval of these connected ideas at test supports recollection during recognition.

Long and Prat (2002) hypothesized that knowledge effects have been underestimated in previous studies of text memory in which recognition was used, because both rec- ollection and familiarity influence the ability to discriminate old from new sentences. They tested this claim in a series of experiments investigating knowledge effects on recognition, using both the remember/know task and the process dissociation procedure, a procedure in which participants associate a test item with its studied context. The participants in their experiments were high or low knowledge about the science fiction saga Star Trek. They read Star Trek and control stories and then made judgments concerning the nature of their memory for sentences. No effect of knowledge was found on overall recognition $\left(d^{\prime}\right)$, replicating the results of previous studies. Long and Prat did find a knowledge effect, however, when they examined recollection and familiarity separately. High-knowledge readers had higher recollection estimates than did lowknowledge ones, but only for Star Trek items.

The importance of background knowledge in understanding a text is acknowledged explicitly in most theories of text comprehension (e.g., Gernsbacher, 1990; Kintsch, 1988). This is not the case for theories of individual differences in reading comprehension. Variation in reading ability is most often attributed to individual differences in reading-specific processes, such as word recognition (Perfetti, 1985, 1994), or individual differences in general cognitive abilities, such as working memory (WM) capacity (Just \& Carpenter, 1992) or inhibition (Gernsbacher \& Faust, 1991, 1995). To the extent that knowledge differences are mentioned at all, they are usually discussed as secondary to other individual-difference factors (see Long, Johns, \& Morris, 2006, for a review).

Our goal in the present study was to investigate the role of background knowledge in recognition memory for text. Specifically, we examined the claim that recollection is a specific marker of the extent to which readers construct discourse models involving the integration of background knowledge with explicit text ideas. Although previous studies by Long and colleagues have shown that prior knowledge is correlated with recollection, they have not shown that variation in background knowledge predicts recollection as strongly, or more strongly, than do other individual-difference factors (Long \& Prat, 2002; Long, Wilson, et al., 2006). Reader characteristics are often correlated; good readers score well on a variety of language and cognitive tests. The influence of background knowledge on recollection may not be strong once shared variance with other reader characteristics is controlled (Long, Wilson, et al., 2006).

We administered tests of language and cognitive abilities to determine whether background knowledge is uniquely related to recollection. We included multiple measures of factors that have been shown to be important in discriminating good adult readers from poor ones (Long, Wilson, et al., 2006). These included measures of word recognition ability, WM capacity, verbal ability (e.g., vocabulary and comprehension tests), and print exposure. We also included measures of reasoning ability to determine whether recollection is related to variation in high-level cognitive ability. If variation in background knowledge is critical in predicting the ability to integrate ideas across sentences, it should be as important in predicting text memory as 
other individual-difference factors. Moreover, individual differences in background knowledge should be related to recollection, not familiarity, to the extent that recollection is a specific marker of processes that occur when text ideas are integrated with relevant world knowledge.

\section{METHOD}

\section{Participants}

The participants were 149 students at the University of California, Davis, who were paid $\$ 10 / \mathrm{h}$. All were native English speakers, and none had a diagnosed reading or learning disability.

\section{Materials and Procedure}

Texts. We selected five texts on topics in biology. Two were articles from the science section of the New York Times. The topics were neurodegenerative disease (771 words) and classification in evolutionary biology (1,625 words). The remaining texts were from the Encyclopedia Britannica. The topics were plant organs (433 words), biochemistry $(1,178)$, and bacteria (1,214 words).

Recognition tests. We constructed a recognition test for each text, consisting of 20 old and 20 new items. Old items consisted of main ideas and details. Main ideas were identified by 15 students who read the texts and highlighted 15 sentences in each that they believed conveyed major ideas. For each text, we selected the 10 sentences with the most participant "votes." Details were selected randomly from sentences that no participant identified as stating a main idea.

We constructed distractor sentences that had considerable overlap in content with information in the texts. This was done in order to address an important limitation in using recognition to study text memory: Recognition of text ideas is very prone to ceiling effects. This is a problem even when old responses are segregated into remember and know judgments. Ceiling effects can be minimized by encouraging participants to adopt relatively stringent response criteria. In the study of text memory, this is usually done by selecting distractor sentences that are similar in content to studied ones (e.g., paraphrases, inferences, unstudied script-typical events).

We used two types of distractors in this study: inferences and foils. Inferences were identified by a second group of 15 students. They read the texts and answered why questions about each sentence. We examined the answers to identify causal inferences, using criteria de- scribed by Graesser and Clark (1985). Foils were selected from texts that were not used in this study but were about the same topics. We selected 10 sentences from each. The foils contained new information, but the information was true about the topic. For example, we identified a text about bacteria that was similar in style and content to the one that we used here. We selected 10 sentences from it, so that each contained information that was not in our original text but was consistent with its content.

The participants received a recognition test after each text. They responded remember if they had a vivid memory of reading the sentence. They responded know if they were confident that they had read the sentence previously but could not recall specific details about it.

Individual-difference tests. We administered tests to assess comprehension ability and four reader characteristics that are typically associated with it (Long, Wilson, et al., 2006): word-decoding skill, WM capacity, print exposure, and knowledge. We also assessed general reasoning ability. We used multiple measures of each characteristic to enhance the reliability of our assessments (Waters \& Caplan, 2003). Our tests included three measures of word recognition skill: (1) naming (latencies to pseudowords, high-frequency words, and low-frequency words), (2) phonological decision (latencies to decide which of two letter strings [e.g., brane, blane] is a real word if pronounced; Bell \& Perfetti, 1994), and (3) orthographic decision (latencies to decide which of two letter strings [brane, brain] is a correctly spelled word; Bell \& Perfetti, 1994). Our tests also included two measures of WM capacity: (1) reading span and (2) operation span (the number of words recalled while sentences are read or arithmetic problems are solved simultaneously; Daneman \& Carpenter, 1980; Engle, Tuholski, Laughlin, \& Conway, 1999). Also included were the vocabulary and comprehension sections of the Nelson-Denny Reading Test (Form H) and two measures of reasoning: (1) Raven's Advanced Progressive Matrices (Raven, 1962) and (2) the GRE analytic section (Educational Testing Service, 1992). Two measures of print exposure were included: (1) the Author Recognition Test and (2) the Magazine Recognition Test (recognition checklists involving discrimination of author names and magazine titles from foils; Stanovich \& West, 1989). Finally, there were two tests of knowledge about biology: (1) the SAT II test in biology and (2) the Scientist Recognition Test (a recognition checklist containing names of prominent historical and contemporary individuals in science, interleaved with distractor names).

Table 1

Matrix Loadings From an Exploratory Factor Analysis Using Maximum Likelihood Estimation and Promax Rotation (Power $=4 ; n=149$ )

\begin{tabular}{|c|c|c|c|c|c|}
\hline \multirow[b]{2}{*}{ Test } & \multicolumn{5}{|c|}{ Factor } \\
\hline & Decoding & $\begin{array}{c}\text { Verbal } \\
\text { Ability }\end{array}$ & $\begin{array}{l}\text { Working } \\
\text { Memory } \\
\text { Capacity }\end{array}$ & Reasoning & Knowledge \\
\hline \multicolumn{6}{|l|}{ Naming } \\
\hline Pseudowords & .95 & .20 & -.04 & .02 & -.01 \\
\hline High-frequency words & .94 & .17 & -.02 & -.08 & -.02 \\
\hline Low-frequency words & .96 & .13 & .01 & -.04 & -.03 \\
\hline Orthographic decision & .62 & .47 & .23 & -.17 & -.09 \\
\hline Phonological decision & .67 & .35 & .31 & .13 & -.16 \\
\hline Operation span & -.15 & .48 & .61 & -.20 & .30 \\
\hline Reading span & -.28 & .28 & .63 & -.39 & .28 \\
\hline Nelson-Denny vocabulary & -.38 & .68 & -.32 & .07 & .01 \\
\hline Nelson-Denny comprehension & -.32 & .55 & -.35 & .05 & -.14 \\
\hline Raven matrices & -.17 & .34 & .37 & .58 & -.25 \\
\hline Analytical reasoning & -.26 & .24 & .19 & .58 & -.31 \\
\hline Author recognition & -.36 & .49 & -.14 & -.07 & .23 \\
\hline Magazine recognition & -.15 & .23 & -.09 & -.22 & .16 \\
\hline Biology & -.05 & .34 & .05 & .10 & .64 \\
\hline Scientist recognition & -.21 & .27 & -.13 & -.06 & .62 \\
\hline
\end{tabular}

Note-The highest factor loading for each measure appears in bold. 
Table 2

Bivariate Correlations Among Composite Measures

\begin{tabular}{lccccc}
\hline \multicolumn{1}{c}{ Measure } & Decoding & $\begin{array}{c}\text { Verbal } \\
\text { Ability }\end{array}$ & $\begin{array}{c}\text { Working } \\
\text { Memory } \\
\text { Capacity }\end{array}$ & Reasoning & Knowledge \\
\hline Decoding & 1.00 & & & & \\
Verbal ability & $-.32^{* *}$ & 1.00 & & & \\
WM capacity & $-.17^{*}$ & $.17^{*}$ & 1.00 & & \\
Reasoning & -.11 & $.38^{* *}$ & $.30^{* *}$ & 1.00 & 1.00 \\
Knowledge & -.03 & $.56^{* *}$ & $.18^{*}$ & $.39^{* *}$ & \\
${ }^{*} p<.05 .{ }^{* *} p<.01$. & & & &
\end{tabular}

The participants received the individual-difference tests across seven sessions. Each session was approximately $1 \mathrm{~h}$. They participated in two additional sessions in which they read the texts and received the recognition tests.

\section{RESULTS}

\section{Factor Analysis}

We analyzed the individual-difference tests to identify reader characteristics for use in subsequent regression analyses. The scree test suggested five factors. These were rotated to the Promax criterion, power $=4$; estimation was maximum likelihood. The factors accounted for $75.2 \%$ of the variance; the loadings appear in Table 1 . The factors reflected the reader characteristics that we described above, except that print exposure was not identified as an independent factor.

We created five composites on the basis of the factor analysis. These were (1) decoding (latencies to pseudowords, high-frequency and low-frequency words, orthographic pairs, and phonological pairs), (2) verbal ability (scores on the Nelson-Denny vocabulary and comprehension subtests and the Author Recognition Test), ${ }^{1}$ (3) WM span (total items recalled on the reading and operation span tests), (4) reasoning (scores on the Raven's Matrices and Analytical Reasoning Tests), and (5) knowledge (scores on the Biology Test and the Scientist Recognition Test). Bivariate correlations among the composites appear in Table 2.

\section{Recognition Results}

We calculated recollection and familiarity estimates for old and new items. Recollection was the probability of a remember response; familiarity was the conditional probability of a know response, given that an item was not recollected $[\mathrm{know} /(1-$ remember $)]$.

Old items. Mean recollection and familiarity estimates for old items appear in Table 3. The regression analyses revealed significant effects on recollection $[F(5,144)=$ $5.77, M S_{\mathrm{e}}=.01, p<.001$, and $F(5,144)=4.03, M S_{\mathrm{e}}=$ $.02, p=.001$, for main ideas and details, respectively]. In contrast, the regression equations for the familiarity estimates were not reliable $\left[F(5,144)=1.26, M S_{\mathrm{e}}=.03\right.$, $p=.28$, and $F(5,144)=0.75, M S_{\mathrm{e}}=.03, p=.61$, for main ideas and details, respectively]. The standardized regression coefficients appear in Table 4. Knowledge about science was the only reliable predictor.

New items. Mean recollection and familiarity estimates for new items (inferences and foils) also appear in Table 3.
With respect to inferences, we found reliable effects on recollection $\left[F(5,144)=7.32, M S_{\mathrm{e}}=.01, p<.001\right]$, but not on familiarity $[F(5,144)=0.59, p=.74]$. The coefficients appear in Table 5. Recollection of inferences decreased as a function of reasoning, verbal ability, and WM capacity. Interestingly, knowledge was positively related to recollection.

Our analyses of the foils yielded reliable effects on both recollection $\left[F(1,144)=3.91, M S_{\mathrm{e}}=.00, p=.001\right]$ and familiarity $\left[F(1,144)=3.32, M S_{\mathrm{e}}=.01, p=.004\right]$. High reasoning scores were associated with low recollection and low familiarity estimates.

\section{DISCUSSION}

We conducted this study in order to investigate the importance of background knowledge in comprehension ability and text memory. In most models of text comprehension, world knowledge is necessary for constructing coherent discourse models - those in which relevant knowledge is integrated with explicit text ideas. Despite the importance of background knowledge in theories of text comprehension, variation in knowledge has played a relatively minor role in theories of individual differences in reading comprehension. We investigated two hypotheses in this study. First, we hypothesized that background knowledge would predict text memory even after controlling for other reader characteristics. Second, we hypothesized that background knowledge would be uniquely related to recollection, because recollection is a specific marker of the processes involved in elaborating a memory trace with preexisting knowledge. Our findings were consistent with both of these hypotheses.

In previous research, recognition has seldom been as effective as recall in detecting variation in readers' text memory. The results of the present study help to explain

Table 3

Mean Recollection and Familiarity Estimates (With Standard Deviations)

\begin{tabular}{cccccc}
\hline & \multicolumn{2}{c}{ Recollection } & & \multicolumn{2}{c}{ Familiarity } \\
\cline { 2 - 3 } \cline { 5 - 6 } Item Type & $M$ & $S D$ & & $M$ & $S D$ \\
\hline Old items & & & & \\
Main ideas & .62 & .17 & .53 & .20 \\
Details & .53 & .16 & .51 & .20 \\
New items & & & & \\
Inferences & .15 & .10 & .36 & .15 \\
Foils & .09 & .07 & .26 & .11 \\
\hline
\end{tabular}


Table 4

Regression Analyses of Recollection and Familiarity Estimates to Old Items

\begin{tabular}{|c|c|c|c|c|c|c|c|c|}
\hline \multirow[b]{3}{*}{ Predictor } & \multicolumn{4}{|c|}{ Main Ideas } & \multicolumn{4}{|c|}{ Details } \\
\hline & \multicolumn{2}{|c|}{ Recollection } & \multicolumn{2}{|c|}{ Familiarity } & \multicolumn{2}{|c|}{ Recollection } & \multicolumn{2}{|c|}{ Familiarity } \\
\hline & $\beta$ & $t$ & $\beta$ & $t$ & $\beta$ & $t$ & $\beta$ & $t$ \\
\hline Decoding & -.08 & -0.88 & .07 & 0.79 & -.02 & -0.24 & .08 & 0.85 \\
\hline Verbal ability & .03 & 0.33 & .27 & $2.12^{*}$ & .13 & 1.26 & .15 & 1.34 \\
\hline Working memory capacity & -.06 & -0.77 & .07 & 0.80 & .10 & 1.13 & .01 & 0.06 \\
\hline Reasoning & .11 & 1.30 & .08 & 0.86 & .13 & 1.45 & .08 & 0.79 \\
\hline Knowledge & .45 & $4.74^{* *}$ & -.10 & -1.00 & .30 & $3.07^{* *}$ & -.01 & -0.12 \\
\hline
\end{tabular}

why. Recognition can be based on familiarity, a process that is relatively unaffected by comprehension-related factors. Even readers who perform poorly on standardized comprehension tests construct accurate representations of individual sentences (Long et al., 1994, 1997). These representations, however, are not sufficient for recall. Readers recall a text only when text ideas are integrated across sentences and are linked to background knowledge. The processes that are involved in making these connections facilitate retrieval in recall and recollection in recognition.

We found that knowledge was the only reliable predictor of recollection for old items. Recollection was unaffected by other comprehension-related factors, such as word decoding and WM capacity. These results suggest that reading skill alone may be insufficient for producing text representations that can be retrieved from memory under a variety of different conditions. When strong retrieval cues are present at test, as they are in recognition, readers can rely on familiarity in accessing their text representations. Familiarity will be less helpful when retrieval cues are minimal; access to the text representation will depend more on the reader's background knowledge than on the reader's overall comprehension ability.

We have argued that background knowledge is important in vivid text memory because the processes involved in integrating text ideas with knowledge yield memory traces for sentences that are accompanied by episodic details during retrieval. These associations are the foundation for remember judgments at test. Another possibility, however, is that knowledge alone gives rise to remember judgments and that memory for the text is irrelevant. We think that this possibility is unlikely, for two reasons. First, our distractor sentences included some that were true about the texts (foils) but were not likely inferences. If readers simply relied on their background knowledge to make recognition judgments, we should have found knowledge effects for the foils. However, knowledge did not predict responses to foils; reasoning was the only reliable predictor. Second, Long and her colleagues have used a task, the process dissociation procedure, to study knowledge effects on recognition (Long \& Prat, 2002; Long, Wilson, et al., 2006). Background knowledge alone cannot be used to perform this task. In their studies, highknowledge and low-knowledge readers read pairs of texts and then received recognition tests in which they had to associate test sentences with the specific texts in which the sentences appeared. The association of test sentence to text could be made only if the readers had memory for the texts; background knowledge alone would not provide the necessary information. The findings with the process dissociation procedure were the same as those with the remember/know task. Knowledge affected recollection estimates but not familiarity estimates.

One surprising finding in this study was the positive relation between background knowledge and recollection of inferences. A possible explanation is that high-knowledge readers made source errors in response to the inferences. That is, they generated the inference either during comprehension or when they encountered the inference at test. They then mistakenly attributed the source of their memory to the text, rather than to their background knowledge. Lowknowledge readers were less likely than high-knowledge ones to make the inference during comprehension or at test; thus, they were less likely to have an illusory experience of recollection to the inference distractors.

We have used the dual-process model as a framework for guiding our hypotheses and interpreting our findings about the relation between knowledge and text memory. The framework underlies our claim that recollection is more sensitive than familiarity to the quality of a reader's discourse

Table 5

Regression Analyses of Recollection and Familiarity Estimates to New Items

\begin{tabular}{|c|c|c|c|c|c|c|c|c|}
\hline \multirow[b]{3}{*}{ Predictor } & \multicolumn{4}{|c|}{ Inferences } & \multicolumn{4}{|c|}{ Foils } \\
\hline & \multicolumn{2}{|c|}{ Recollection } & \multicolumn{2}{|c|}{ Familiarity } & \multicolumn{2}{|c|}{ Recollection } & \multicolumn{2}{|c|}{ Familiarity } \\
\hline & $\beta$ & $t$ & $\beta$ & $t$ & $\beta$ & $t$ & $\beta$ & $t$ \\
\hline Decoding & -.15 & -1.76 & .04 & 0.45 & -.01 & -0.10 & -.03 & -0.37 \\
\hline Verbal ability & -.35 & $-3.68^{* *}$ & .07 & 0.60 & -.18 & -1.75 & -.15 & -1.42 \\
\hline Working memory capacity & -.16 & $-2.06^{*}$ & .10 & 1.16 & -.05 & -0.61 & -.03 & -0.32 \\
\hline Reasoning & -.30 & $-3.49^{* *}$ & -.14 & -1.49 & -.30 & $-3.36^{* *}$ & -.22 & $-2.43^{*}$ \\
\hline Knowledge & .19 & $2.03^{*}$ & -.00 & -0.02 & .07 & 0.72 & -.08 & -0.80 \\
\hline
\end{tabular}

${ }^{*} p<.05 . \quad{ }^{* *} p<.01$. 
model. This claim, however, does not depend on the validity of the dual-processing framework. Single-process models have been proposed that attribute recollection to the retrieval of strong, content-rich representations and familiarity to the retrieval of weak, less specific ones (e.g., Donaldson, 1996). These models explain our data equally well. According to this view, the processes that are involved in constructing discourse models yield content-rich representations, and these representations support recollection at test.

In conclusion, knowledge about the topic of a text leads to vivid memory for text ideas and accounts for unique variance in recollection. High-knowledge readers report vivid memories for old sentences (those that appeared in the text), as well as vivid memories for some new sentences. These "false memories," however, are not errors in the usual sense of the term; they occur only in response to sentences that are identified as inferences. Thus, these errors are systematically related to the reader's ability to construct a discourse model and, like the recollection of old ideas, reflect the importance of background knowledge in text memory.

\section{AUTHOR NOTE}

The research reported here was supported by grants from the National Science Foundation (NSF0231152) and the National Institutes of Health (NICHD 1 R01 HD48914-1A2). Correspondence concerning this article should be sent to D. L. Long, Department of Psychology, University of California, One Shields Avenue, Davis, CA 95616 (e-mail: dllong@ ucdavis.edu).

\section{REFERENCES}

Alba, J. W., \& Hasher, L. (1983). Is memory schematic? Psychological Bulletin, 93, 203-231.

Anderson, R. C., \& Pichert, J. W. (1978). Recall of previously unrecallable information following a shift in perspective. Journal of Verbal Learning \& Verbal Behavior, 17, 1-12.

Bell, L. C., \& Perfetti, C. A. (1994). Reading skill: Some adult comparisons. Journal of Educational Psychology, 86, 244-255.

Bower, G. H., Black, J. B., \& TuRner, T. J. (1979). Scripts in memory for text. Cognitive Psychology, 11, 177-220.

BRANSFord, J. D., \& Johnson, M. K. (1972). Contextual prerequisites for understanding: Some investigations of comprehension and recall. Journal of Verbal Learning \& Verbal Behavior, 11, 717-726.

Daneman, M., \& CARPenter, P. A. (1980). Individual differences in working memory and reading. Journal of Verbal Learning \& Verbal Behavior, 19, 450-466.

DonalDSON, W. (1996). The role of decision processes in remembering and knowing. Memory \& Cognition, 24, 523-533.

Engle, R. W., Tuholski, S. W., Laughlin, J. E., \& Conway, A. R. A. (1999). Working memory, short-term memory, and general fluid intelligence: A latent-variable approach. Journal of Experimental Psychology: General, 128, 309-331.

GeRNSBACHER, M. A. (1990). Language comprehension as structure building. Hillsdale, NJ: Erlbaum.

Gernsbacher, M. A., \& FAuST, M. E. (1991). The mechanism of suppression: A component of general comprehension skill. Journal of Experimental Psychology: Learning, Memory, \& Cognition, 17, 245-262.

Gernsbacher, M. A., \& Faust, M. E. (1995). Skilled suppression. In F. N. Dempster \& C. J. Brainerd (Eds.), Interference and inhibition in cognition (pp. 295-327). San Diego: Academic Press.
Graesser, A. C., \& Clark, L. F. (1985). Structures and procedures of implicit knowledge. Norwood, NJ: Ablex.

JACOBY, L. L., \& DALLAS, M. (1981). On the relationship between autobiographical memory and perceptual learning. Journal of Experimental Psychology: General, 110, 306-340.

Johnson, W., \& Kieras, D. (1983). Representation-saving effects of prior knowledge in memory for simple technical prose. Memory \& Cognition, 11, 456-466.

Just, M. A., \& CARPENTER, P. A. (1992). A capacity theory of comprehension: Individual differences in working memory. Psychological Review, 99, 122-149.

KINTSCH, W. (1988). The role of knowledge in discourse comprehension: A construction-integration model. Psychological Review, 95, 163-182.

Long, D. L., Johns, C. L., \& MorRis, P. E. (2006). Comprehension ability in mature readers. In M. J. Traxler \& M. A. Gernsbacher (Eds.), Handbook of psycholinguistics (2nd ed., pp. 801-834). Amsterdam: Elsevier.

Long, D. L., Oppy, B. J., \& Seely, M. R. (1994). Individual differences in the time course of inferential processing. Journal of Experimental Psychology: Learning, Memory, \& Cognition, 20, 1456-1470.

LONG, D. L., OpPY, B. J., \& SEELY, M. R. (1997). Individual differences in readers' sentence- and text-level representations. Journal of Memory \& Language, 36, 129-145.

Long, D. L., \& Prat, C. S. (2002). Memory for Star Trek: The role of prior knowledge in recognition revisited. Journal of Experimental Psychology: Learning, Memory, \& Cognition, 28, 1073-1082.

Long, D. L., Wilson, J., Hurley, R., \& Prat, C. S. (2006). Assessing text representations with recognition: The interaction of domain knowledge and text coherence. Journal of Experimental Psychology: Learning, Memory, \& Cognition, 32, 816-827.

Mandler, G. (1980). Recognizing: The judgment of previous occurrence. Psychological Review, 87, 252-271.

MoravcsiK, J. E., \& KinTSCH, W. (1993). Writing quality, reading skills, and domain knowledge as factors in text comprehension. Canadian Journal of Experimental Psychology, 47, 360-374.

Perfetti, C. A. (1985). Reading ability. New York: Oxford University Press.

Perfetti, C. A. (1994). Psycholinguistics and reading ability. In M. A. Gernsbacher (Ed.), Handbook of psycholinguistics (pp. 849-894). San Diego: Academic Press.

RAVEN, J. C. (1962). Advanced progressive matrices (Set II). London: Lewis.

Stanovich, K. E., \& West, R. F. (1989). Exposure to print and orthographic processing. Reading Research Quarterly, 24, 402-433.

Sulin, R. A., \& Dooling, D. J. (1974). Intrusion of a thematic idea in retention of prose. Journal of Experimental Psychology, 103, 255-262.

Tulving, E. (1985). Memory and consciousness. Canadian Psychology, 26, 1-12.

Waters, G. S., \& CAPLAN, D. (2003). The reliability and stability of verbal working memory measures. Behavior Research Methods, Instruments, \& Computers, 35, 550-564.

YonELINAS, A. P. (2002). The nature of recollection and familiarity: A review of 30 years of research. Journal of Memory \& Language, 46, 441-517.

YonelinAS, A. P., \& JACOBY, L. L. (1995). The relation between remembering and knowing as bases for recognition: Effects of size congruency. Journal of Memory \& Language, 34, 622-643.

\section{NOTE}

1. We did not include the Magazine Recognition Test in our composite measures, because it did not load highly on any of the factors.

(Manuscript received July 20, 2007; revision accepted for publication January 30 , 2008.) 